\title{
High Level of miR-18a Promote Radiotherapy Progress in Non-Small Cell Lung Cancer
}

\author{
Dachuan H*, Xiaobo Z, Pengli W, Zujun Y and \\ Xue L \\ Department of Respiratory, Ziyang Municipal People's \\ Hospital, Ziyang 641300, Sichuan, China \\ *Corresponding author: He Dachuan, MD, \\ Department of Respiratory, Ziyang Municipal People's \\ Hospital, Ziyang 641300, Sichuan, China
}

Received: August 11, 2021; Accepted: September 27, 2021; Published: October 04, 2021

\begin{abstract}
Palliative radiotherapy has become an important treatment way for local control of advanced Non-Small Cell Lung Cancer (NSCLC). Current investigation aims to clarify miR-18a as a predictor of radiotherapy sensitivity, its cutoff value for predicting response. 70 patients with NSCLC were enrolled for radiotherapy during the period of 2018-2020. The level of miR-18a in blood were detected by using Quantitative PCR. Another seven variances including age, sex, smoking history, pathologic stage, radiation therapy days, radiation dosage and radiation type were enrolled to predict radiation therapy response. The level of hasmiR-18a showed higher in patients with poor effects after radiation therapy $(\mathrm{P}<0.001)$. The optimal cut-off value of 18.287 for miR-18a alone with AUC of $0.879(95 \%$ confidence interval, $0.8-0.958)$ can predict the radiation therapy effectiveness. Hsa-mir-18a is significantly positive $(\beta 1=-0.2845, P=0.0001)$ in logistical regression model AUC of 0.89 (95\% confidence interval, 0.811-0.97). Hsa-mir-18a is the most important factors in random forest model with an AUC of 0.69 (95\% confidence interval, $0.6-0.82)$ for predicting the radiation therapy effectiveness. High expressed hsa-mir-18a positively correlated with radiation therapy progress in patients with advanced NSCLC.
\end{abstract}

Keywords: miR-18a; Radiotherapy; NSCLC

\section{Introduction}

Lung cancer is one of the most common malignant tumors, and approximately 1.5 million people die from lung cancer every year in the world [1]. Non-Small Cell Lung Cancer (NSCLC) accounts for more than $80 \%$ of all lung cancers [2]. Approximately $75 \%$ of lung cancer patients are already in advanced stage (inoperable stage IIIA, IIIB and IV) at the time of clinical diagnosis [3]. According to guidelines recommendation of metastatic NSCLC, the treatment principle of advanced NSCLC is systemic treatment supplemented by palliative radiotherapy [4]. Palliative radiotherapy has become an important treatment way for local control of advanced NSCLC, because some patients are not suitable for EGFR-TKI treatment, or tolerate chemotherapy or concurrent chemoradiation [5].

At present, there is no uniform standard for the radiation dose of palliative radiotherapy, and the range of dose reported in published literature is 1-60 Gy [6]. The NSCLC guidelines recommend that higher doses or longer courses of radiotherapy (such as greater than $30 \mathrm{~Gy} / 10$ times) are associated with moderate survival and symptom improvement for palliative radiotherapy accompany with chest symptoms [4]. But increasing dose of radiotherapy will increase the adverse reactions. More importantly, some patients are effective while others are ineffective under the same therapeutic dose, and even some patients endured serious side effects [7]. Therefore, for palliative radiotherapy of advanced NSCLC, one of the most effective strategies is to maximize the killing of tumor cells while having fewer adverse reactions, so as to achieve a balance of effectiveness and safety [8]. If the patient's sensitivity to radiation can be predicted before treatment, then an appropriate radiation dose can balance effectiveness and side effects. However, at present, there is no marker that can be used to predict the sensitivity of radiotherapy in clinical practice. Therefore, finding some biomarkers related to radiotherapy sensitivity has important for improving and guiding clinical NSCLC radiotherapy.

miR-18a belongs to the miR-17-92 cluster which was partly regulated by the oncogenic transcription factor c-Myc [9]. The oncogenic role of the miR-17-92 cluster has also been well-recorded which associated with tumor proliferation and progression. Shen et al. found that the over expression of miR-18a was strongly correlated with tumor differentiation, regional lymph node metastasis and clinical TNM stage in NSCLC [10]. In addition, previous studies showed that miR-18a plays a key role in development of colon cancer, breast cancer and prostate cancer. The mechanism behind miR-18a inducing cell proliferation was stimulating cyclin D1 via the PTENPI3K-AKT-mTOR signaling axis. Moreover, miR-18a expression also accelerates cell invasion, promotes $\mathrm{G} 1 / \mathrm{S}$ phase cell cycle arrest in NSCLC. Therefore, miR-18a level may be associated with therapeutic response, and miR-18a downregulation sensitized NSCLC cells to radiation treatment.

Current investigation aims to clarify miR-18a as a predictor of radiotherapy sensitivity, its cutoff value for predicting response. The circulating miR-18a levels have been refereed as novel and promising prognostic biomarkers in patients with NSCLC [11]. It is proposed that patients with advanced NSCLC who meet the indications for radiotherapy alone will be designed with a radiotherapy plan based on the size of the lung lesions [12]. miR-18a predicts radiation sensitivity cutoff value, adverse reactions, quality of life, and explore the correlation between the dynamic changes of miR-18a and objective response rate. The research results are expected to verify the correlation between lung cancer radiotherapy sensitivity and plasma 
miR-18a, and provide molecular markers for predicting the efficacy of NSCLC radiotherapy sensitivity, so that patients with advanced NSCLC can obtain the best treatment plan, thereby improving the quality of life of patients and prolonging survival time.

\section{Materials and Methods}

\section{Samples}

All patients with NSCLC were enrolled from the Ziyang Municipal People's Hospital during the period of 2018-2020. Diagnostic evaluations were performed separately in patients by using standardized criteria for diagnosing NSCLC [4]. Patient inclusion criteria: 1) All patients were diagnosed by histopathological and/or cytological examination; 2) Patient's physical condition shows good; 3) Estimated survival time more than 3 months; 4) Age showed among 40 90 years old; 5) The physiological functions of heart, liver, lung, kidney and bone marrow are basically normal; 6) Patient have not received radiotherapy for primary tumors and metastases before; 7) Patients receive at least 2-4 cycles of cisplatin-based chemotherapy. If the disease does not progress after chemotherapy, radiotherapy will be started 2 weeks after the last chemotherapy; 8 ) Patients show EGFR-sensitive mutants, but refused targeted therapy, or undergo targeted therapy for drug resistance; 9) Volunteer to join the investigation, have good compliance, can cooperate with the observation, and sign a written informed consent. Exclusion criteria: 1) Patients with severe dysfunction of vital organs (heart, liver, kidney); 2) Patients with pregnancy, other malignant tumors, or a history of autoimmune diseases; 3 ) Patients with acute infectious disease or chronic infectious disease; 4) Patients with history of drug allergy or allergic constitution; 5) Patients who participate in other clinical trials at the same time. Patients with Complete Response (CR) after radical radiotherapy and patients who achieved Partial Response (PR) or relapsed within six months were compared each other. This study was conducted with the approval of the ethics committee of Ziyang Municipal People's Hospital. All participants gave written informed consent.

\section{Quantitative PCR}

Peripheral blood $(5 \mathrm{ml})$ was collected from involved participants, and genomic DNA was isolated from peripheral and total RNA was extracted from blood using TRIzol reagent (Takara Biotechnology Co., China) and cDNA was synthesized by PrimeScript ${ }^{\mathrm{TM}} \mathrm{RT}$ reagent kit (Takara Biotechnology Co., Ltd.), according to the manufacturer's instructions. Quantitative RT-PCR was performed with a StepOnePlus Real-Time PCR system (Invitrogen Life Technologies, Carlsbad, CA, USA) using SYBR-Green Real-time PCR Master mix (Toyobo, Osaka, Japan). Circularization of linear miRNAs is followed by rolling circle amplification during the reverse transcription. Control reactions were conducted using the same amount of RNA without reverse transcription. The primer was purchased from sigma (\#lot: MIRAP00034-250RXN). Has-miR-18a primer listed as following, F: 5'-G C T A A G G T G C A T C T A G T G C A G A-3', R: 5'-T C G T A G G C A A T T C G T T T T T T T T T T T T T T T T T T T T C T A T-3'. Expression of each gene was normalized to GAPDH. F: 5'-G G C C C C T C T G G A A A G C T G T G-3', R: 5'-C C A G G C G G C A T G T C A G A T C-3'.

\section{Variance and statistical analysis}

Except the level of has-miR-18a in patient blood, another seven variances including age, sex ( $1=$ male; $2=$ female), smoking history (1=Lifelong Non-smoker; $2=$ Current smoker; $3=$ Current reformed smoker for $>15$ years; $4=$ Current reformed smoker for $\leq 15$ years), pathologic stage ( $1=$ stage I, $2=$ stage II, $3=$ stage III), radiation therapy days (range: 1 76 days), radiation dosage (range: 100 8200 cgy) and radiation type ( $1=$ exterl beam; $2=$ interl beam) were enrolled to predict radiation therapy response. Primary observation endpoint events include stable and progress after radiation therapy.

Clinical characteristics results were presented as the means \pm standard deviation (SD). Parametric and non-parametric test were used to analysis numeric variable as appropriate. Categorical variables were compared by using Chi-square test or Fisher exact test as appropriate. $\mathrm{P}<0.05$ was considered statistically significant. Part of the plots were conducted by using GraphPad Prism 8.4. All statistical analyses were conducted using $\mathrm{R}$ software (http://www.R-project. org/). Bonferroni correction was used for the analysis of contingency tables, depending on the sample size.

\section{Results}

70 patients with NSCLC accepted radiation therapy were enrolled
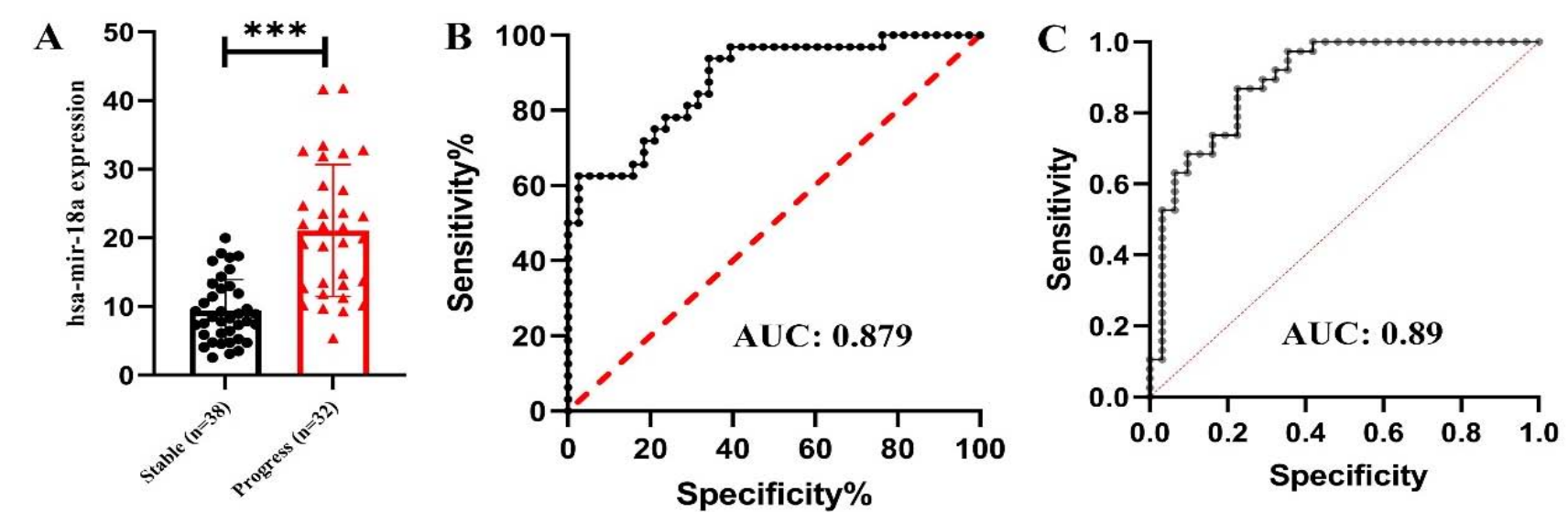

Figure 1: A) Comparison level of microRNA has-miR-18a between stable and progress patients after radiation therapy. B) The results of the ROC curve analysis of the miR-18a assay for detecting radiation therapy response. C) The results of the ROC curve analysis of using logistical regression prediction value to detect radiation therapy response. 
Table 1: Clinical characteristics of NSCLC patients after radiation therapy.

\begin{tabular}{|c|c|c|c|c|}
\hline & Stable $(n=38)$ & Progress $(n=32)$ & Value & $\mathbf{P}$ \\
\hline Age (Years) & $68.05 \pm 9.957$ & $66.06 \pm 11.22$ & $0.786(t)$ & 0.482 \\
\hline Sex (Male/Female) & $17 / 21$ & $17 / 15$ & $0.21\left(x^{2}\right)$ & 0.646 \\
\hline Radiation days & $1 \sim 76$ & $1 \sim 50$ & 571.5 (Mann-Whitney U) & 0.669 \\
\hline Pathology Stages (I/II/III) & $23 / 8 / 7$ & $17 / 8 / 7$ & $4.56\left(x^{2}\right)$ & 0.102 \\
\hline Radiation dosage (cgy) & $4172 \pm 1793$ & $3793 \pm 1925$ & $0.85(t)$ & 0.398 \\
\hline Radiation type (exterl/interl) & $36 / 2$ & $25 / 7$ & (Fisher exact test) & 0.07 \\
\hline Smoking history & $9 / 6 / 9 / 14$ & $6 / 10 / 7 / 9$ & $2.44\left(X^{2}\right)$ & 0.486 \\
\hline
\end{tabular}

t: Student $t$ test; $X^{2}$ : Chi square test; Cgy: Centigray.

A

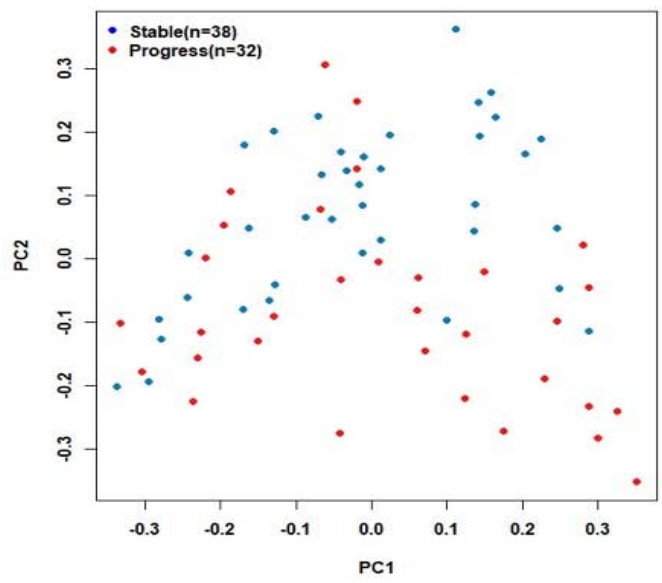

C

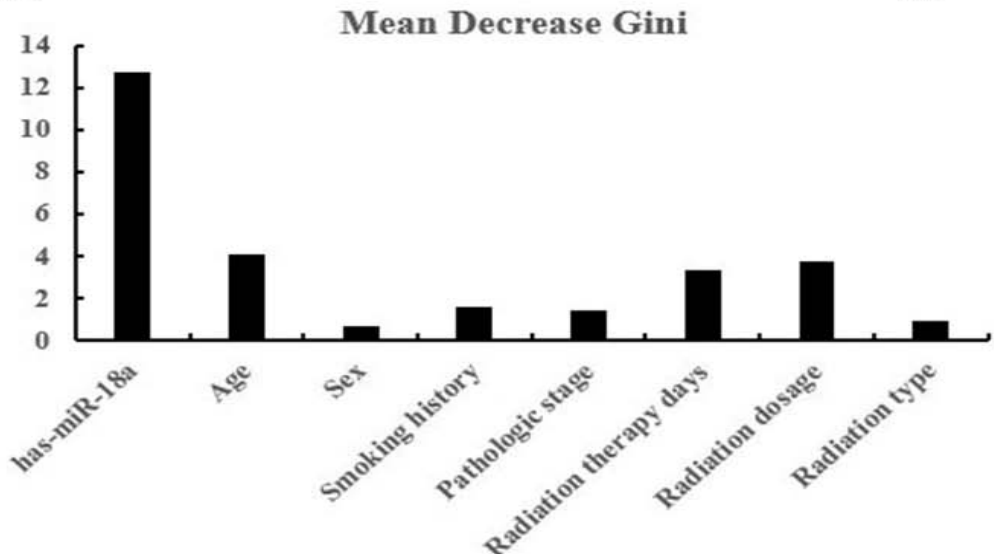

B
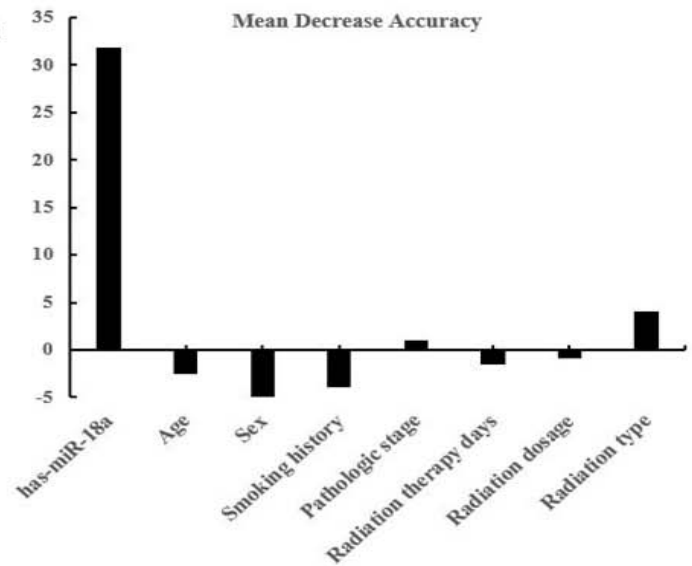

$\mathbf{D}$

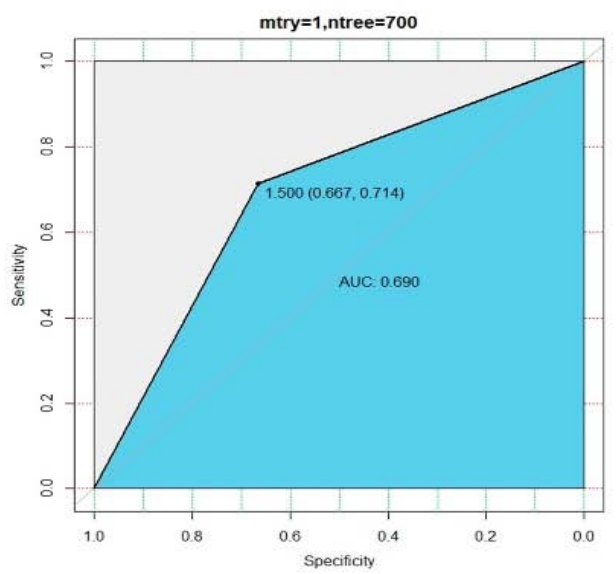

Figure 2: A) PCA analysis of clinical characterizes between stable and progress patients after radiation therapy. B) Mean decrease accuracy in predicting radiation therapy response by using random forest model. C) Mean decrease gini in predicting radiation therapy response by using random forest model. D) The results of the ROC curve analysis of using random forest prediction value to detect radiation therapy response.

in current investigation. No significant difference was found between baseline clinical characteristics (Table 1). However, the level of hasmiR-18a showed higher in patients with poor effects after radiation therapy (Figure $1 \mathrm{~A}, \mathrm{P}<0.001$ ). The analysis revealed that at the optimal cut-off value of 18.287 for miR-18a, the sensitivity was $62.5 \%$ and the specificity was $97.4 \%$, with an AUC of 0.879 (95\% confidence interval, 0.8-0.958) (Figure 1B).

Next, the factors that influence effects of radiation therapy are investigated. Table 2 reports the logistic regression results of equation below table, using response results as the instrument of smoothing.
Current model tests the effects of ownership variables such as hsamir-18a, Age, sex and Radiation type. The estimated coefficients indicate that hsa-mir-18a is significantly positive $(\beta 1=-0.2845$, $\mathrm{P}=0.0001$ ). The ROC analysis revealed AUC of 0.89 (95\% confidence interval, 0.811-0.97) (Figure 1C).

Principal component analysis, as an unsupervised learning method couldn't distinguish effects for patients enduring radiation therapy (Figure 2A). Random forest, as a classification algorithm were employed in current data analysis. Classification accuracy of $\mathrm{RF}$ method depends on user-defined parameters $\mathrm{N}$ and $\mathrm{m}$, whereas 
Table 2: Logistical regression results for predicting response of radiation therapy.

\begin{tabular}{|c|c|c|c|c|}
\hline & Variable & Estimate & Standard error & z value \\
\hline$\beta 0$ & Intercept & 3.5 & 3.292 & 1.063 \\
\hline$\beta 1$ & hsa-mir-18a & -0.2845 & 0.07402 & 3.844 \\
\hline$\beta 2$ & Age & 0.007692 & 0.04194 & 0.1834 \\
\hline$\beta 3$ & Sex (Female) & -0.7464 & 0.8006 & 0.9323 \\
\hline B4 & Radiation type (interl) & -1.98 & 1.554 & 0.3512 \\
\hline
\end{tabular}

Equation: Response $\sim \beta 0+\beta 1^{*}$ hsa-mir-18a $+\beta 2^{*}$ Age $+\beta 3^{*}$ Sex $+\beta 4^{*}$ Radiation type

Tjur's R-squared: 0.484; AIC: 83.95; Multiple comparisons correction: none.

$\mathrm{N}=700$ and $\mathrm{m}=2$ is selected for optimal radiation therapy effects prediction. The OOB estimates of error rate 0.27 were used to measure the variance importance of patients developed from radiation therapy. Mean decreased accuracy and Gini were demonstrated in Figure $2 \mathrm{~B}$ and $2 \mathrm{C}$, and the level of hsa-mir-18a indicated as the most important variance for detecting radiation therapy response. The analysis revealed that at the optimal cut-off value of 1.5 for random forest model, the sensitivity was $66.7 \%$ and the specificity was $71.4 \%$, with an AUC of 0.69 (95\% confidence interval, 0.6-0.82) (Figure 2D).

\section{Discussion}

In current studies, the level of miR-18a in patient with NSCLC were detected for predicting effects from radiotherapy. The result showed that whether miR-18a alone, logistical regression model or random forest model support that miR-18a can predict the radiation therapy effectiveness.

miR-18a located in the $13 \mathrm{q} 31.1$ chromosome region, where it was regulated by the oncogenic transcription factor c-Myc [13]. Previous studies demonstrated that the miR-18a cluster playing an oncogenic role is highly expressed in several types of cancers, such as lung cancer, gastric cancer, melanoma, breast cancer, and so on $[14,15]$. Krysan et al. proved that prostaglandin E2, rich in NSCLC and inflammatory cells in the tumor microenvironment, can drive cell proliferation and promote resistance to pharmacologically induced apoptosis in a c-Myc and miR-17-92-dependent manner [16]. In current investigation, the level of miR-18a positively correlated with radiation therapy progress in patients with advanced NSCLC and this finding taken more interesting. A separate study reported earlier that miR-18a increases lung cancer cell growth which making it a more dependable therapeutic strategy. In clinical researches, miR18a expression strongly correlated with clinical TNM stage, tumor differentiation and even regional lymph node metastasis in NSCLC [17]. The mechanism behind miR-18a in the development of tumor mainly lies in many aspects. Liang et al. found that miR-18a-5p could drive cancer by directly targeting IRF2, and might also have a close correlation between the p53 and NF- $\mathrm{kB}$ signaling pathway [18]. In addition, $\mathrm{Yu}$ et al. demonstrated that miR-18a upregulation induces cell proliferation by stimulating cyclin D1 via the PTEN-PI3KAKT-mTOR signaling axis in breast cancer [19]. Furthermore, high miR-18a expression was correlated with a high recurrence rate in hepatocellular carcinoma by promoting pathological angiogenesis via an increase in VEGFA expression [20]. To test the relationship between miR-18a expression and radiotherapeutic efficacy, the level of miR-18a was detected. The results showed that miR-18a was significantly associated with therapeutic response, exhibiting higher expression level in non-responsive patients. In actually, the expression levels of miR-18a were also up-regulated in A549 lung cancer cell line which accelerates cell invasion, promotes G1/S phase cell cycle arrest and enhances the action of pro-apoptotic agents [21]. More interesting, the depletion of miR-18a increased A549 cell radiosensitivity [21]. Whether miR-18a alone, logistical regression model or random forest model indicate higher confidence of miR$18 \mathrm{a}$ in predicting radiosensitivity. Current studies have revealed that miR-18a is a promising biomarker in NSCLC radiosensitivity, due to its key role in cancer development. Therefore, miR-18a may be as a potent drug target for NSCLC. Although the mechanism remains unclearly, pathway analysis indicated that the direct gene targets are associated mainly with the VEGFA pathway, et al. These studies provide a new method of drug design and a powerful application of miRNAs in cancer treatment.

\section{Conclusion}

In conclusion, our study was to confirm the effect of miR-18a overexpression on the radiosensitivity of NSCLC. High expressed hsa-mir-18a positively correlated with radiation therapy progress in patients with advanced NSCLC.

\section{Declaration}

Funding: The project was supported by grants from Ziyang municipal Bureau of science and technology (Zykjjsc20-yyjc-2019-07).

Ethics approval: All patients signed written informed consent for the use of the anonymized data for research or educational purpose.

\section{References}

1. Islami F, Torre LA, Jemal A. Global trends of lung cancer mortality and smoking prevalence. Translational lung cancer research. 2015; 4: 327.

2. Goldstraw $P$, Ball $D$, Jett JR, Le Chevalier T, Lim E, Nicholson AG, et al. Nonsmall-cell lung cancer. The Lancet. 2011; 378: 1727-1740.

3. Barr Kumarakulasinghe N, Zanwijk Nv, Soo RA. Molecular targeted therapy in the treatment of advanced stage non-small cell lung cancer (NSCLC). Respirology. 2015; 20: 370-378.

4. Reck M, Popat S, Reinmuth N, De Ruysscher D, Kerr K, Peters S. Metastatic non-small-cell lung cancer (NSCLC): ESMO Clinical Practice Guidelines for diagnosis, treatment and follow-up. Annals of oncology. 2014; 25: 27-39.

5. Yang C, Sun B, Wang J, Meng X, Xuan L, Zhang J, et al. Outcomes and toxicity of stereotactic body radiation therapy for advanced stage ultra-central non-small cell lung cancer. Thoracic Cancer. 2019; 10: 1567-1575.

6. Luo H-S, Huang H-C, Lin L-X. Effect of modern high-dose versus standarddose radiation in definitive concurrent chemo-radiotherapy on outcome of esophageal squamous cell cancer: a meta-analysis. Radiation Oncology. 2019; 14: 1-9.

7. Trapani S, Manicone M, Sikokis A, D'Abbiero N, Salaroli F, Ceccon G, et al. Effectiveness and safety of "real" concurrent stereotactic radiotherapy and immunotherapy in metastatic solid tumors: A systematic review. Critical 
reviews in oncology/hematology. 2019; 142: 9-15.

8. van Diessen JN, Kwint M, Sonke J-J, Walraven I, Stam B, de Langen AJ, et al. Safety and efficacy of reduced dose and margins to involved lymph node metastases in locally advanced NSCLC patients. Radiotherapy and Oncology. 2020; 143: 66-72.

9. Mihailovich M, Bremang M, Spadotto V, Musiani D, Vitale E, Varano G, et al miR-17-92 fine-tunes MYC expression and function to ensure optimal B cell lymphoma growth. Nature communications. 2015; 6: 8725.

10. Shen Z, Wu X, Wang Z, Li B, Zhu X. Effect of miR-18a overexpression on the radiosensitivity of non-small cell lung cancer. International journal of clinical and experimental pathology. 2015; 8: 643-648.

11. Xu X, Zhu S, Tao Z, Ye S. High circulating miR-18a, miR-20a, and miR-92a expression correlates with poor prognosis in patients with non-small cell lung cancer. Cancer medicine. 2018; 7: 21-31.

12. Bodor JN, Boumber Y, Borghaei H. Biomarkers for immune checkpoin inhibition in non-small cell lung cancer (NSCLC). Cancer. 2020; 126: 260270 .

13. Siavrienè E, Preikšaitienè E, Maldžienè Ž, Mikštienè $V$, Rančelis $T$, Ambrozaitytè $L$, et al. A de novo 13q31. 3 micro duplication encompassing the miR-17-92 cluster results in features mirroring those associated with Feingold syndrome 2. Gene. 2020: 144816.

14. Komatsu S, Ichikawa D, Takeshita H, Morimura R, Hirajima S, Tsujiura M, et al. Circulating miR-18a: a sensitive cancer screening biomarker in human cancer. In vivo. 2014; 28: 293-297.
15. Sanchez-Mejias A, Kwon J, Chew XH, Siemens A, Sohn HS, Jing G, et al. A novel SOCS5/miR-18/miR-25 axis promotes tumorigenesis in liver cancer International journal of cancer. 2019; 144: 311-321.

16. Krysan K, Kusko R, Grogan T, O'Hearn J, Reckamp KL, Walser TC, et al. PGE2-driven expression of c-Myc and oncomiR-17-92 contributes to apoptosis resistance in NSCLC. Molecular Cancer Research. 2014; 12: 765774.

17. Hayashita $Y$, Osada H, Tatematsu $Y$, Yamada H, Yanagisawa K, Tomida $\mathrm{S}$, et al. A polycistronic microRNA cluster, miR-17-92, is overexpressed in human lung cancers and enhances cell proliferation. Cancer research. 2005: 65: 9628-9632.

18. Liang C, Zhang X, Wang H-M, Liu X-M, Zhang X-j, Zheng B, et al. MicroRNA18a-5p functions as an oncogene by directly targeting IRF2 in lung cancer. Cell death \& disease. 2017; 8: e2764.

19. Yu Z, Wang C, Wang M, Li Z, Casimiro MC, Liu M, et al. A cyclin D1/microRNA $17 / 20$ regulatory feedback loop in control of breast cancer cell proliferation. The Journal of cell biology. 2008; 182: 509-517.

20. Sun J, Lu H, Wang $X$, Jin H. MicroRNAs in hepatocellular carcinoma: regulation, function, and clinical implications. The Scientific World Journal. 2013; 2013.

21. Xiao H, Liu Y, Liang P, Wang B, Tan H, Zhang $Y$, et al. TP53TG1 enhances cisplatin sensitivity of non-small cell lung cancer cells through regulating miR18a/PTEN axis. Cell \& bioscience. 2018; 8: 1-13. 